\title{
The history of dipper functions
}

\author{
Joshua A. Solomon \\ City University, London, England
}

\begin{abstract}
Dipper-shaped curves often accurately depict the relationship between a baseline, or "pedestal," magnitude and a just noticeable difference in it. This tutorial traces the 45 -year history of the dipper function in auditory and visual psychophysics, focusing on when they happen and why. Popular theories of both positive and negative masking (i.e., the "handle" and "dip," respectively) are described. Sometimes, but not always, negative masking disappears with an appropriate redescription of stimulus magnitude.
\end{abstract}

\section{Gaussian Noise}

Did you ever have your walls dusted? I did, and the duster (who shall remain nameless) managed to knock all of my pictures out of alignment. You can try, but it is impossible to hang a picture perfectly straight. Sometimes the right side seems a little too high, sometimes the left. I would change my mind about the tilt without even moving the picture. This uncertainty is manifest in psychometric functions for orientation discrimination. An example is shown in Figure 1, which summarizes observer M.M.'s responses to visual targets having different tilts. Consider the large data point: When forced to choose, observer M.M. said "clockwise" on 14 of 58 trials in which the true stimulus orientation was $1^{\circ}$ anticlockwise of vertical.

Notice that the psychometric function is well fit by a Gaussian distribution. That is,

$$
\Psi(\theta) \approx \Phi(\theta / \sigma),
$$

where $\Phi$ is the normal cumulative distribution function $(\mathrm{CDF})$ and $\sigma=1.2^{\circ}$. This fit suggests a Gaussian source of noise, intrinsic to the visual system, which corrupts the ability to estimate orientation.

\section{Discriminating Different Levels of Gaussian Noise}

Although persuasive, this suggestion is at odds with the way my pictures used to look - that is, perfectly straight. If the apparent orientation of each picture was corrupted by Gaussian noise, then how could they ever have seemed to be aligned? This puzzle prompted Morgan, Chubb, and Solomon $(2008)^{1}$ to speculate that maybe the visual system squelches its own noise. To test this possibility, they measured how well observers could discriminate between two textures whose otherwise parallel elements were tilted with different amounts of Gaussian noise.
Figure $2 \mathrm{~A}$ shows an example of their stimuli. The observers had a two-alternative forced choice (2AFC): They were shown two textures and asked to select the one having greater variance. This variance was manipulated until observers were responding with an accuracy of $82 \%$.

The horizontal position of each point in Figure 2B shows the standard deviation of orientations in the less variable, or "pedestal," texture. The vertical position of each point shows how much greater the standard deviation of the other texture needed to be for M.M. to identify it with $82 \%$ accuracy. This is the JND, or just noticeable difference, in standard deviation. The JNDs form a dipper-shaped function of pedestal magnitude; that is, as the pedestal increases from zero, the JNDs first decrease and then increase. This result may seem counterintuitive, but dipper functions of this general shape, with a "dip" in the middle and a "handle" on the right, are ubiquitous in contemporary psychophysics. The historical review that follows should help to steer interested empiricists away from particularly contentious issues.

\section{Weber's Law}

Dipper functions are a subset of those functions that describe the relationship between $I$, a baseline magnitude, ${ }^{2}$ and $\Delta I_{\mathrm{JND}}$, a just noticeable increment. An even simpler relationship, attributed to Weber (by Fechner, 1860/1912), can be written

$$
\Delta I_{\mathrm{JND}} / I=k,
$$

where the "Weber fraction" $k$ does not vary with $I$. So much has been written about Weber's law (and the "nearmisses" thereto) that I feel unable to add anything intelligent on the topic. Instead, I direct the reader to chapter 1 of Laming (1986). His chapter 2 contains an unbeatable review of the psychophysical methods appropriate for obtaining Weber's law. 


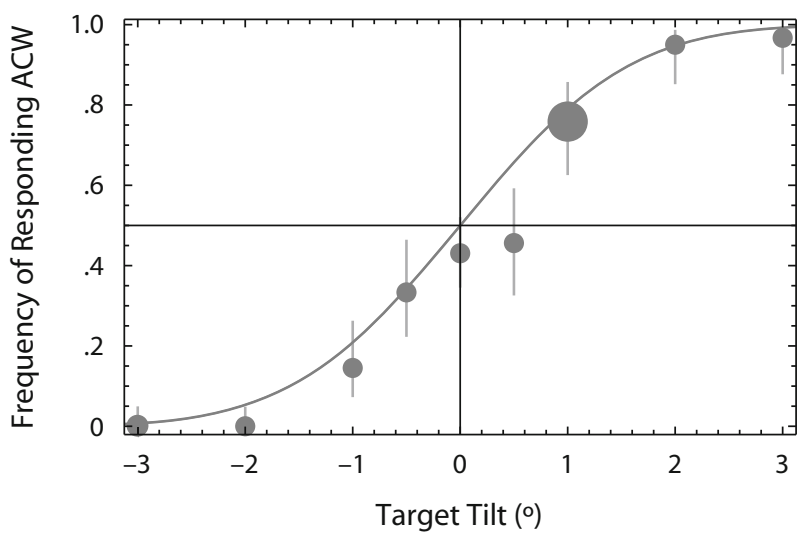

Figure 1. A psychometric function for orientation discrimination. Each point shows how frequently M.M. responded "anticlockwise" (ACW) in Phase 1 of Solomon, Felisberti, and Morgan (2004; cf. their Figure 3, which shows data from Phase 2). Negative values indicate clockwise tilts. Error bars reflect $95 \%$ confidence intervals for the true probabilities. The solid curve is Equation 1.

Conventional psychophysical methods (e.g., 2AFC) constrain the possible relationships between $I$ and $\Delta I_{\mathrm{JND}}$. Recent discussions of these constraints can be found in Doble, Falmagne, and Berg (2003) and Iverson (2006a, 2006b). All of the constraints are satisfied by Weber's law.

\section{Luminance Discrimination}

Following many previous authors, Bartlett (1942) obtained Weber fractions for detecting luminance increments on a steady background. He obtained different Weber fractions when $I$ and $I+\Delta I$ were presented on a background $\neq I$. Like many other authors, Bartlett used the same symbol to denote both an arbitrary increment and a just noticeable one. In this discussion, I will reserve $\Delta I$ for the former and $\Delta I_{\mathrm{JND}}$ for the latter.

Following Bartlett (1942), Cornsweet and Pinsker (1965) distinguished between 2AFC detection of luminance increments on a steady background and $2 \mathrm{AFC}$ discrimination of luminance "pulses" on a dark background. Only the latter type of experiment produced dippershaped plots of $\log \Delta I_{\mathrm{JND}}$ versus $\log I$ (cf. Blackwell, 1963); these dipper functions had long, straight handles with a gradient of 1 . Thus, Weber's law held for large values of $I$ - that is, whenever the pulses could be easily detected.

Plotting tip: I recommend using coordinate systems in which a log unit has the same length vertically and horizontally. This makes it relatively easy to visually estimate the handle gradient, and thus the validity of Weber's law for large values of $I$.

\section{Variance Discrimination}

The very first dipper function was published by Raab, Osman, and Rich (1963b), showing data from detecting brief increments in the amplitude of continuous auditory noises of various intensities. Since loud background noises usually make it hard to hear quiet increments, the effect of background noise is often called "masking." Raab et al. (1963b) coined the term "negative masking" to describe how the power of the just detectable increment decreases as the background noise increases from zero. The funny thing is that Raab et al. (1963b) did not even do the experiment themselves; the data had originally been published by Miller in 1947.

Miller (1947) plotted the just detectable increment in power, instead of the power of the just detectable increment, and consequently did not get any negative masking. This distinction is important, so I will explain why no increase in the just detectable increment in power can actually be a decrease in the power of the just detectable increment.

Here, knowing a bit of the physics of signal detection will be useful. Random fluctuations in pressure sound like noise, and the power of auditory noise is proportional to the variance in pressure. The amplitude of an auditory noise is typically expressed as the root-mean-squared (RMS) pressure; that is, the amplitude is proportional to the square root of power. Finally, a quantity we will discuss later is energy. To get any signal's energy, you need to integrate its power over both its extent (in time, for auditory signals; in space and time, for visual ones) and its bandwidth.

Now, consider the case of detection - that is, in the case of no background noise, which is what the 0 under "Power of Background" means in the first row of Table 1. When the power is zero, the amplitude is also zero. Without loss of generality, we can assume that a short burst of noise requires an RMS amplitude of $\sigma$ to be just detected in total quiet. The symbol $\sigma$ is typically used to denote the standard deviation of a Gaussian distribution, and that is how the amplitude of Gaussian noise is typically defined - as the standard deviation of pressure.

The total amplitude of a just detectable noise burst is thus $0+\sigma=\sigma$, and since power is proportional to pressure variance, the total power at detection threshold is some constant $a$ times $\sigma^{2}$. To calculate the just noticeable increase in power, we subtract the background power from this value $\left(a \sigma^{2}-0=a \sigma^{2}\right)$, and the power of the just noticeable increment is the same constant $a$ times the same variance $\sigma^{2}$.

Moving on to the bottom row of Table 1, we now consider a just detectable background. Its amplitude, as we have already assumed, will be at least approximately identical to that of the just detectable noise burst, $\sigma$, and its power is thus $a \sigma^{2}$.

Here is where the calculations get interesting. Assume that this very faint background produces no change in the just noticeable increment in power, which is what Miller (1947) reported. Previously, we had a just noticeable increase in power of $a \sigma^{2}$, so here we will also have a just noticeable increase in power of $a \sigma^{2}$. The total power is the power of the background plus the just noticeable increase in power, so that is $a \sigma^{2}+a \sigma^{2}=2 a \sigma^{2}$. Since the total power must be $a$ times the total amplitude squared, the total amplitude must be $(\sqrt{2}) \sigma$. The just noticeable increment in amplitude is the difference between the total amplitude and the amplitude of the background alone. 
A
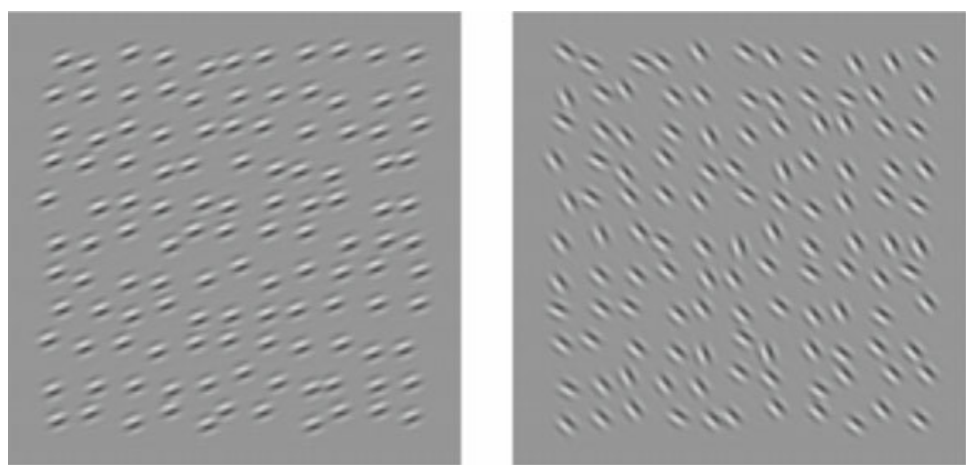

B

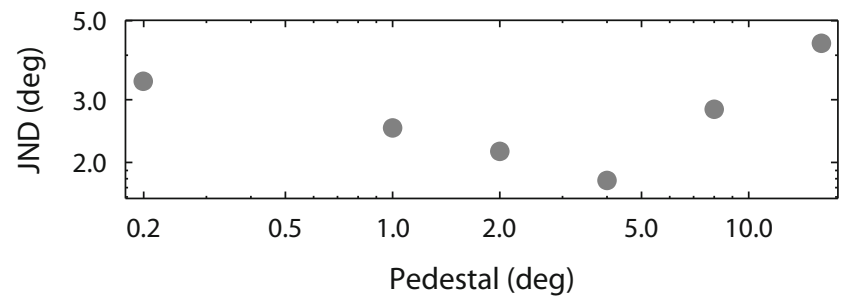

Figure 2. Example stimuli and example results from Morgan et al. (2008). The observers' 2 AFC task was to report which of two images had higher variability in orientation. In the example here (panel $\mathrm{A}$ ), there is zero variability in the image on the left. Orientations on the right were sampled from a Gaussian distribution with an $8^{\circ}$ standard deviation. Each point in panel B shows observer M.M.'s (82\% correct) just noticeable difference (JND) in the standard deviation.

That is, $(\sqrt{ } 2) \sigma-\sigma=(\sqrt{2}-1) \sigma \approx 0.414 \sigma$, and at last we can calculate the power of the just noticeable increment. This is simply $a$ times the square of the preceding value: $a(0.414 \sigma)^{2}=0.172 a \sigma^{2}$, which is a fraction of the power of a just detectable noise.

The preceding example shows why Raab et al. (1963b) seemed to get "negative masking" when they replotted Miller's (1947) data as the power of the just noticeable increment. This leads us to the question, what if I replotted Morgan et al.'s (2008) data in a similar way? That is, what if I plotted the just noticeable differences in variance, rather than the just noticeable differences in standard deviation?

The answer appears in Figure 3. Here are the same data as in Figure 2, replotted in terms of orientation variance. ${ }^{3}$ In this case, there is almost no evidence of any negative masking. The take-home message from this exercise is that, in some cases, negative masking can disappear with an alternative description of stimulus magnitude.
Green (1960a) formulated the ideal observer for detecting various signals. He showed that when the signal is a sample of noise, the ideal observer is an energy detector. It is clear, from his Equation 7b, that Weber's law is a consequence of this optimal detector for a noise signal.

However, human observers are not ideal for a variety of reasons. Typically, they are inefficient, which means that they are unable to utilize all of the available information when making a decision. For example, when estimating signal variance, human observers must rely on a limited sample, and that sample may get corrupted by sensory noise. I fit a noisy, inefficient version of Green's (1960a) idealobserver model to Morgan et al.'s (2008) discriminations of orientation variance. There are two free parameters in this model: the standard deviation of the putatively Gaussian noise that corrupts each estimate of orientation, and the number of Gabor patterns whose orientations the observers

Table 1

Negative Masking With a Constant JND in Power

\begin{tabular}{|c|c|c|c|c|c|c|}
\hline $\begin{array}{c}\text { RMS } \\
\text { Amplitude } \\
\text { of } \\
\text { Background }\end{array}$ & $\begin{array}{c}\text { Power } \\
\text { of } \\
\text { Background }\end{array}$ & $\begin{array}{c}\text { Just } \\
\text { Noticeable } \\
\text { Increment in } \\
\text { Amplitude }\end{array}$ & $\begin{array}{c}\text { Total } \\
\text { Amplitude }\end{array}$ & $\begin{array}{c}\text { Total } \\
\text { Power }\end{array}$ & $\begin{array}{c}\text { Just } \\
\text { Noticeable } \\
\text { Increase in } \\
\text { Power }\end{array}$ & $\begin{array}{l}\text { Power of } \\
\text { the Just } \\
\text { Noticeable } \\
\text { Increment }\end{array}$ \\
\hline 0 & 0 & $\sigma$ & $\sigma$ & $a \sigma^{2}$ & $a \sigma^{2}$ & $a \sigma^{2}$ \\
\hline$\sigma$ & $a \sigma^{2}$ & $0.414 \sigma$ & $(\sqrt{2}) \sigma$ & $2 a \sigma^{2}$ & $a \sigma^{2}$ & $0.172 a \sigma^{2}$ \\
\hline
\end{tabular}




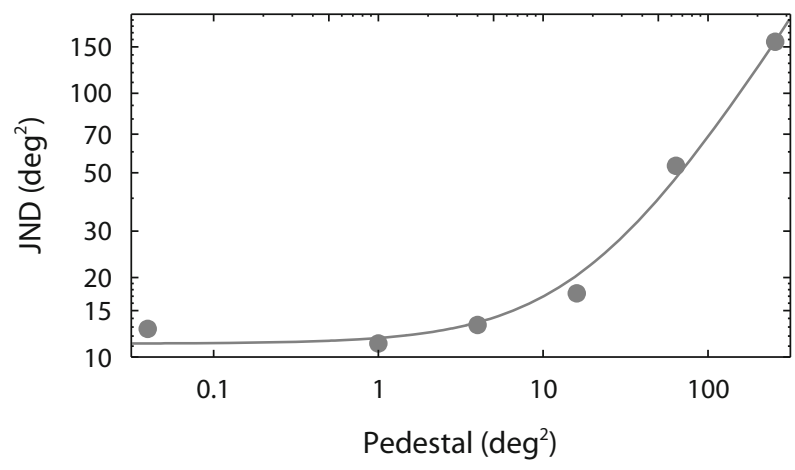

Figure 3. Replotted results from Morgan et al. (2008). Here, the vertical axis describes the just noticeable difference (JND) in orientation variance. The solid curve illustrates the performance of a two-parameter observer, whose otherwise ideal decision is based on a limited number (19) of Gaussian-noise-perturbed $\left(\sigma=4.4^{\circ}\right)$ samples in each image. These two parameter values minimize the RMS error in log degrees squared (cf. the analysis of Morgan et al., who maximized the likelihood of their psychometric data).

estimated in each texture - that is, the sample size. The fit to M.M.'s data is shown as the solid curve in Figure 3.

One conclusion to draw from this high-quality fit is that acuity for variance is best described in terms of variance. Maybe this isn't very surprising, but as will be discussed below, the appropriate scale for other acuities is not always this obvious. Note here that I am using the word "acuity" in its most general sense, to refer to an observer's sensitivity to fine detail in the size or strength of some stimulus.

Before turning away from the dipper function for orientation variance, I must mention its similarity to the dipper function for blur discrimination (Watt \& Morgan, 1983). Morgan et al. (2008) noted that just as the sum of two independent sources of noise (e.g., that in the stimulus and one intrinsic to the visual system) can be determined by convolving their probability density functions, the combined effects of stimulus and intrinsic blurs can be determined by convolving their individual point-spread functions. Thus, the dipper functions for Gaussian blur and for orientation variance may be modeled by the same noisy observer.

\section{Amplitude Discrimination: Appropriate Description}

Although Raab et al. (1963b) were the first to use the term "negative masking," it was Green (1960b) who first reported evidence of it, when he measured how easy it was to detect changes in the amplitude of a pure tone. Specifically, a faint tone was easier to detect when it was presented in phase with another, near-threshold tone of the same frequency and duration. Pfafflin and Mathews (1962) introduced the term "pedestal" to describe Green's (1960b) facilitating tone. ${ }^{4}$ Although the energy detector is not ideal for discriminating between tone amplitudes, Pfafflin and Mathews found that Green's (1960b) results with faint tones were nonetheless consistent with its behavior.

Because of the energy model's success in explaining Green's (1960b) negative masking and psychometric slopes,
Raab, Osman, and Rich (1963a) argued that discrimination results should be described and plotted in terms of energy differences rather than amplitude differences. Laming (1986, p. 13) also reviewed the problem of the appropriate scale for dipper functions, but he came to the opposite conclusion. One of his arguments for amplitude is that amplitude frequently has a more "natural" unit of measurement (cf. degrees squared, which seems less natural).

Weber's law itself can supply an even better reason. Fechner (1860/1912) and many others believed that the intensity of sensory experiences increased in proportion to the ratio $\Delta I / I$. The accumulation of sensory noise should produce Gaussian perturbations in these intensities. Thus, psychometric functions of this ratio should resemble the Gaussian CDF-that is, $\psi(\Delta I / I) \approx \Phi(\Delta I / I)$. According to Laming (1986), this prediction is borne out when the pedestal $I$ is easily detected and $\Delta I$ is proportional to an amplitude difference, not to a power or energy difference. Of course, Laming (1986) did not examine every type of acuity, and there may be some exceptions. Orientation variance may be one of these exceptions - we do not know, because Morgan et al. (2008) used a staircase procedure to quickly estimate JNDs.

Methodological tip: Try to collect complete psychometric functions, so that their shapes can be compared with the Gaussian CDF.

\section{Amplitude Discrimination: Shape of the Dipper Function}

Hanna, von Gierke, and Green (1986) obtained dipper functions for the $2 \mathrm{AFC}$ amplitude discrimination of short tones. When these tones were presented against a background of auditory noise, their data were like those of Morgan et al. (2008): Negative masking did not survive the transformation into energy differences. ${ }^{5}$ However, when the tones were presented against a quiet background, Hanna et al. found that negative masking did survive the transformation. The implications of this result appear below.

Craig (1974) obtained a dipper function for discriminating the amplitudes of vibrotactile stimulation. The handle had a gradient of 1 . I replotted his JNDs as energy differences and found that one point still strongly suggested negative masking.

A huge number of studies have reported dipper functions for the detection of contrast increments in sinusoidal luminance gratings. The first of these was performed by Campbell and Kulikowski (1966). They used the method of adjustment and plotted their dipper function upsidedown with respect to current conventions. The handle of their dipper function had a gradient of -1 , seemingly consistent with Weber's law.

Using the 2AFC paradigm, Nachmias and Sansbury (1974) published the next major study on contrast discrimination. I transformed their data into energy differences and found only one point that still suggested negative masking. This data point may be an outlier. Legge and Foley (1980) conducted a much larger study using a similar methodology, but none of their dipper functions suggest negative masking when replotted in 
terms of energy differences. Legge himself (Legge \& Viemeister, 1988) was one of the first to realize how the dipper function for contrast changes shape when replotted as a dipper function for contrast energy. However, even in today's literature, most contrast dippers appear as Nachmias and Sansbury's did: JNDs in contrast versus pedestal contrast.

Nachmias and Sansbury's (1974) study begat an even more heated controversy, because unlike in the findings of Campbell and Kulikowski (1966), the handle of their dipper function had a gradient $<1$, seemingly inconsistent with Weber's law. Kulikowski and Gorea (1978) responded with evidence suggesting that Campell and Kulikowski's otherwise irreproducible finding could be attributed to contrast adaptation, a by-product of the method of adjustment. However, Legge (1981) failed to replicate Kulikowski and Gorea using 2AFC. He speculated that their results (and Campbell and Kulikowski's) might have been due to some other artifact of the method of adjustment.

\section{Explaining the Dip: Sensory Thresholds}

Now it is time to consider those cases in which negative masking does not disappear when the data are plotted in terms of variance (or power or energy) differences. As noted above, Cornsweet and Pinsker (1965) obtained dipper functions when $I$ was the luminance ${ }^{6}$ of a flash against a dark background and $\Delta I_{\mathrm{JND}}$ was a just noticeable increment in that luminance. The ideal discriminator would consequently produce a monotonically increasing curve like the one in Figure 3. Something else must have been responsible for the dip.

Crozier (1950) speculated that the retina might absorb some quanta that could not be used for vision until yet more were absorbed. In this "photosensitization" process, the retina acts like a kind of watering can, but light goes into it instead of water. Only when the pot is already "full" does additional light produce any output. This sensory threshold theory predicts negative masking even when $\Delta I_{\mathrm{JND}}$ represents an energy difference. Morgan et al.'s (2008) experiment was designed to reveal the existence of an analogous threshold for orientation variance. Although the evidence of negative masking in Figure 3 is meager, a significantly better fit can be made to the raw data summarized therein by bolting a sensory threshold onto the otherwise merely noisy and inefficient observer. (Details appear in Morgan et al., 2008.)

As the name suggests, high-threshold models have sensory thresholds so high that they prevent observers from ever perceiving visual noise. This type of model can be rejected on the basis of Swets, Tanner, and Birdsall's (1961) two-response 4AFC experiment. Without visible noise, unlucky guesses are the only way to make errors in a forced choice experiment. High-threshold models predict that all 4AFC errors would thus be caused by unlucky guesses. Contrary to this idea, Swets et al. found that second responses were more often correct than could be accounted for by guessing. Thus, some $4 \mathrm{AFC}$ errors must have been due to visual noise.

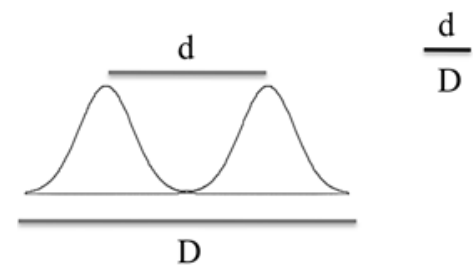

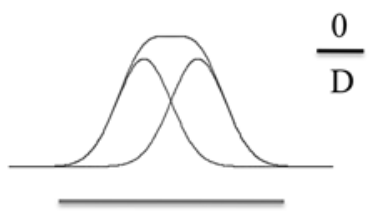

D

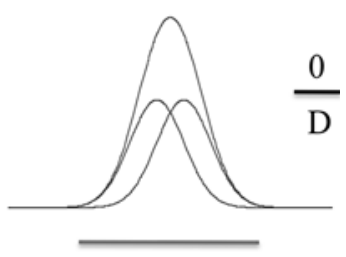

D
Figure 4. Hypothetical computation of spatial intervals. Each panel shows two Gaussian density functions, each of which represents a blurred point of light, and their sum. If separation acuity depends on the ratio of the distance between the peaks in this sum (d) to its overall width (D), the just noticeable differences for large pedestals, which produce bimodal sums, may be smaller than those for small pedestals, which do not.

Green and Swets (1966) identified one low-threshold theory that was not inconsistent with the extant data. As its name suggests, this theory supposes that visual noise is sometimes visible, and thus causes some forced choice errors. All other errors are caused by unlucky guesses. Although Green and Swets did not attempt to fit Swets et al.'s (1961) data with this model, I did (in Solomon, 2007b). ${ }^{7}$ The fit was not very good.

Nonetheless, sensory thresholds might arise naturally in certain situations. One of these is spatial interval discrimination (as noted by David Burr, in his review of the MS. of this article), in which what is measured is the acuity for the distance between two points of light. The idea is that each point gets blurred by the visual system and that acuity might depend on the ratio of the distance between the peaks of the blur circles to the distance between the far edges of the blur circles (see Figure 4). When the points are sufficiently close, there is zero distance between the peaks of the blur circles, and thus they must be moved farther apart for their separation to be detected. (Dipper functions for this task and for a variety of alternative models are discussed in Levi, Jiang, \& Klein, 1990.)

\section{Explaining the Dip: Intrinsic Uncertainty}

Green (1961) described the intrinsic-uncertainty model of pure-tone detection. The idea was initially published by Tanner (1961), but Green (1961) was the first to actually specify the model. Basically, the idea is that observers monitor many irrelevant channels when trying to detect a weak stimulus (see Figure 5). Since these channels play no role in suprathreshold discrimination, detection (i.e., the case in which $I=0$ ) effectively becomes harder.

Intrinsic uncertainty is a powerful theory because, with just two free parameters, it adequately predicts the 


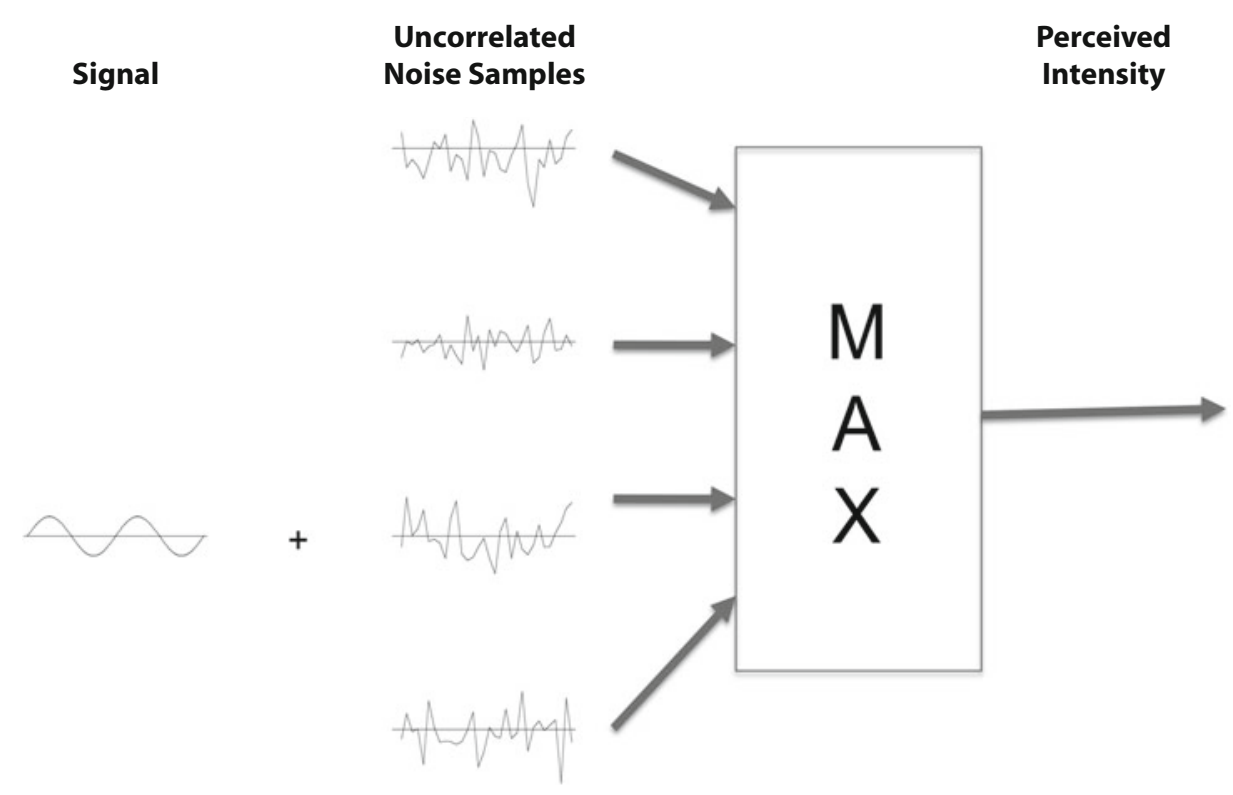

Figure 5. Intrinsic-uncertainty theory. Observers monitor many irrelevant channels (thousands can be required to fit some data; see Pelli, 1985) when trying to detect a weak stimulus.

amount of negative masking, the second-response accuracy in Swets et al.'s (1961) 4AFC detection experiment (Solomon, 2007b), and the slope of the psychometric function for detection. When plotted on a logarithmic abscissa, this slope is completely determined by the number of irrelevant channels. The theory's other free parameter is the amount of noise in each channel. Despite its power, intrinsic uncertainty remains unpopular. One reason for this is Watson, Franks, and Hood's (1972) finding that psychometric functions for detecting pure tones in quiet are even steeper than those for detecting pure tones in noise. Legge, Kersten, and Burgess (1987) replicated Watson et al.'s study in the visual domain and found that the addition of noise, which should not affect intrinsic uncertainty, nonetheless caused the slope of the psychometric function for detection to flatten. Consequently, they rejected intrinsic uncertainty.

\section{Explaining the Dip: Nonlinear Transduction}

Taking their cue from Fechner, Nachmias and Sansbury (1974) suggested that JNDs simply reflect the perceived magnitude of a stimulus. Consequently, they inferred an accelerating transducer for small stimulus contrasts. In particular, they modeled the intensity of the "internal effect" as a power function of stimulus contrast, with an exponent between 2.2 and 2.9, depending on the observer. As

\section{Dipper Function}

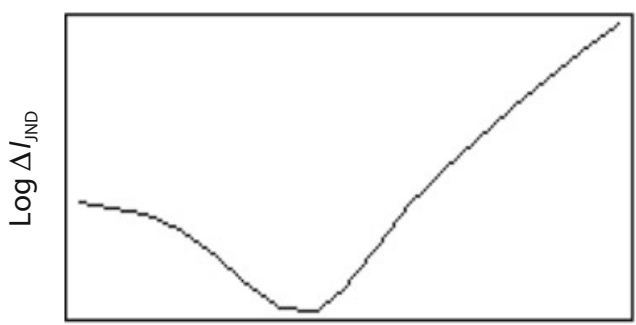

$\log I$
Transducer

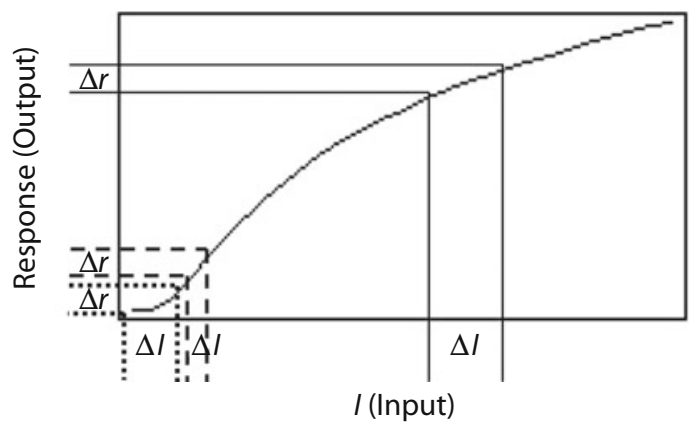

Figure 6. Nonlinear transduction. The transducer panel on the right shows the relationship between stimulus magnitude (on an arbitrary dimension) and the response of one sensory mechanism. In general, two stimuli can be discriminated if and only if the responses they elicit differ by more than some criterion $\Delta r$. Negative masking (indicated by the dipper function on the left) happens whenever the sensory response increases faster than the stimulus magnitude. Consider what happens when the pedestal is zero: The just noticeable difference (JND) for detection is represented as the distance between the two vertical dotted lines. The vertical dashed lines are closer together, and thus the JND for small pedestals is smaller. Because the thin solid lines are farther apart, the JND for large pedestals is larger. 
the pedestal increases, so does the slope of this transducer, and consequently less of an increment in contrast is required for a significant difference in perceived contrast.

For a concrete example, assume that $2.5 \%$ is the necessary contrast for detecting some stimulus (with, e.g., $75 \%$ accuracy in a 2AFC experiment). Without loss of generality, we can say that the $2.5 \%$ stimulus creates an internal effect that is $\Delta r$ greater than that created by a stimulus with $0 \%$ contrast. Now suppose further experimentation reveals that otherwise identical stimuli with $2 \%$ and $3 \%$ contrasts can be similarly discriminated (i.e., with $75 \%$ accuracy in a $2 \mathrm{AFC}$ experiment). Thus, the $3 \%$ stimulus must have created an internal effect $\Delta r$ greater than that created by the $2 \%$ stimulus. Note that a plot of JND versus pedestal contrast would thus suggest negative masking. If we wanted to ascribe this dip to a power-law transducer, we would simply have to find the exponent $p$ that solves the simultaneous equations

$$
0.025^{p}-0=\Delta r=0.03^{p}-0.02^{p} .
$$

In this case, $p \approx 2.5$.

Legge and Foley (1980) also fit their data with a nonlinear transducer model, a modification of the version proposed by Stromeyer and Klein (1974). With four parameters, they showed that it was possible to describe the entire dipper function (see Figure 6).

\section{One-Mechanism Explanations of the Handle}

Thus, the simplest explanation of any dipper function is that it reflects the nonlinear transduction of stimulus magnitude into a sensory response. However, it could be argued that the transducer is not much of an explanation, simply an alternative way to describe the data.

Signal detection theory revolutionized psychophysical theory with its chief implication that all sensory signals are stochastic. To fully account for their 4AFC results, Swets et al. (1961) proposed that the variance of sensory signals increases faster than their means. Pfafflin and Mathews (1962) recognized that this increasing noise had the potential to explain why Weber's law was at least approximately correct even for amplitude discriminations between nonstochastic stimuli. Nonlinear transduction was not necessary.

Legge et al. (1987) argued that compressive-transduction and increasing-noise models are generally equivalent. In other words, we could not confidently infer the transducer function from a dipper function unless we knew the relationship between the sensory response and its variance. And we could also not confidently infer the latter relationship from a dipper unless the transducer were known. Catch-22. Legge et al.'s argument, however, included certain assumptions about the distribution of sensory noise and the form of the psychophysical task, which has left room for more recent attempts to differentiate between the two ideas. In the words of Georgeson and Meese (2006), "the jury's still out." Other significant contributions to this ongoing debate may be found in García-Pérez and AlcaláQuintana (2007); Gorea and Sagi (2001); Katkov, Tsodyks, and Sagi (2006a, 2006b); Klein (2006); Kontsevich, Chen, and Tyler (2002); and Solomon (2007a).

\section{Multiple-Mechanism Explanations of the Handle}

Green (1983) was the first to articulate the suspicion that dipper functions are not necessarily indicative of any simple mapping between a stimulus and a single sensory response, however noisy that response might be. He reported the results of a number of experiments, all of which suggest that amplitude discriminations are based on spectral shape (i.e., a "profile analysis" of the output from narrowly tuned channels) whenever possible (cf. Cornsweet and Pinsker's [1965] interpretations from "pulses" in the dark and Hanna et al.'s [1986] from short tones in quiet). In one of these experiments, later adapted for vision by Nachmias (1993), the intensity of a noise masker varied from interval to interval (i.e., even within a single trial) in the range $30 \pm X \mathrm{~dB} .{ }^{8}$ The question here was how the JND for a pure tone depended on $X$. When each interval was of sufficient length (i.e., $115 \mathrm{msec}$ ), the answer was "not much." As $X$ increased from 0 to 20, the JND increased just $1 \mathrm{~dB}$.

Laming (1986, 1988a, 1988b) proposed that all discriminations are effectively based on the spatiotemporal derivative of stimulus magnitude, rather than on stimulus magnitude itself. (Some discriminations may be based on the second derivative.) When combined with an early source of Poisson (i.e., increasing) noise (e.g., that in the photon flux), this "differential coupling" effectively turns all magnitude discriminations into variance discriminations, and, as shown by Green (1960a), ideal discriminations of variance obey Weber's law. In the studies above, Laming showed how noisy differential coupling, when combined with early noise and half-wave rectification, can produce an approximate square-law transformation of low-intensity sensory input. Negative masking is one result of this accelerating transduction. Possibly the most contentious aspect of Laming's model is his attribution of all suprathreshold visual discrimination errors to quantal fluctuations.

\section{Conclusion}

Most dipper functions really aren't that mysterious, since the dip goes away when the data are replotted on arguably more appropriate axes. If the dip survives this transformation, it strongly suggests some kind of suboptimal processing. Possible explanations of this suboptimality include a sensory threshold, intrinsic uncertainty, nonlinear transduction, or a combination of the three. Morgan et al. (2008) looked for such suboptimality but found only scant evidence for it. The question then remains, why do we not usually notice the visual system's intrinsic noise? If the visual system does ordinarily squelch its own noise (e.g., by applying a sensory threshold), it certainly does not do so when observers are actively trying to detect a stimulus that has been designed to imitate the noise.

\section{AUTHOR NOTE}

This article improved because of close reading by David Burr and Charles Chubb. J.A.S. receives support from Cognitive Systems Foresight Project Grant BB/E000444/1 and Grant EP/E064604 from the Engineering and Physical Sciences Research Council of Great Britain. Correspondence related to this article may be sent to J. A. Solomon, 
Department of Optometry and Visual Science, City University, London EC1V 0HB, England (e-mail: j.a.solomon@city.ac.uk).

\section{REFERENCES}

BARTLETT, N. R. (1942). The discrimination of two simultaneously presented brightnesses. Journal of Experimental Psychology, 31, 380392.

BLACKWELL, H. R. (1963). Neural theories of simple visual discriminations. Journal of the Optical Society of America, 53, 129-160.

CAmpbell, F. W., \& KulikowsKi, J. J. (1966). Orientational selectivity of the human visual system. Journal of Physiology, 187, 437-445.

Cornsweet, T. N., \& Pinsker, H. M. (1965). Luminance discrimination of brief flashes under various conditions of adaptation. Journal of Physiology, 176, 294-310.

CRAIG, J. C. (1974). Vibrotactile difference thresholds for intensity and the effect of a masking stimulus. Perception \& Psychophysics, 15, 123-127.

Crozier, W. J. (1950). On the visibility of radiation at the human fovea. Journal of General Physiology, 34, 87-136.

Doble, C. W., Falmagne, J.-C., \& Berg, B. G. (2003). Recasting (the near-miss to) Weber's law. Psychological Review, 110, 365-375.

FechneR, G. T. (1912). Elements of psychophysics. In B. Rand (Ed.), The classical psychologists: Selections illustrating psychology from Anaxagoras to Wundt (pp. 562-572). Boston: Houghton Mifflin. (Original work published 1860)

García-PÉrez, M. A., \& AlCalá-Quintana, R. (2007). The transducer model for contrast detection and discrimination: Formal relations, implications, and an empirical test. Spatial Vision, 20, 5-43.

Georgeson, M. A., \& Meese, T. S. (2006). Fixed or variable noise in contrast discrimination? The jury's still out. . . . Vision Research, 46, 4294-4303.

Gorea, A., \& SAGI, D. (2001). Disentangling signal from noise in visual contrast discrimination. Nature Neuroscience, 4, 1146-1150.

GreEn, D. M. (1960a). Auditory detection of a noise signal. Journal of the Acoustical Society of America, 32, 121-131.

GreEn, D. M. (1960b). Psychoacoustics and detection theory. Journal of the Acoustical Society of America, 32, 1189-1203.

GreEn, D. M. (1961). Detection of auditory sinusoids of uncertain frequency. Journal of the Acoustical Society of America, 33, 897-903.

GreEn, D. M. (1983). Profile analysis: A different view of auditory intensity discrimination. American Psychologist, 38, 133-142.

Green, D. M., \& Swets, J. A. (1966). Signal detection theory and psychophysics. New York: Wiley.

Hanna, T. E., von Gierke, S. M., \& Green, D. M. (1986). Detection and intensity discrimination of a sinusoid. Journal of the Acoustical Society of America, 80, 1335-1340.

IVERSON, G. J. (2006a). Analytical methods in the theory of psychophysical discrimination I: Inequalities, convexity and integration of just noticeable differences. Journal of Mathematical Psychology, 50, 271-282.

Iverson, G. J. (2006b). Analytical methods in the theory of psychophysical discrimination II: The near-miss to Weber's law, Falmagne's law, the psychophysical power law and the law of similarity. Journal of Mathematical Psychology, 50, 283-289.

KatKov, M., TsodyKs, M., \& SAGi, D. (2006a). Analysis of a twoalternative force-choice signal detection theory model. Journal of Mathematical Psychology, 50, 411-420.

Katkov, M., Tsodyks, M., \& Sagi, D. (2006b). Singularities in the inverse modeling of $2 \mathrm{AFC}$ contrast discrimination data. Vision Research, 46, 259-266.

KLeIN, S. A. (2006). Separating transducer non-linearities and multiplicative noise in contrast discrimination. Vision Research, 46, 42794293.

Kontsevich, L. L., Chen, C.-C., \& Tyler, C. W. (2002). Separating the effects of response nonlinearity and internal noise psychophysically. Vision Research, 42, 1771-1784.

Kulikowski, J. J., \& GoreA, A. (1978). Complete adaptation to patterned stimuli: A necessary and sufficient condition for Weber's law for contrast. Vision Research, 18, 1223-1227.

Laming, D. (1986). Sensory analysis. London: Academic Press.
Laming, D. (1988a). Précis of Sensory analysis. Behavioral \& Brain Sciences, 11, 275-296.

LAMING, D. (1988b). A reexamination of Sensory analysis. Behavioral \& Brain Sciences, 11, 316-336.

LEGGE, G. E. (1981). A power law for contrast discrimination. Vision Research, 21, 457-467.

LEGGE, G. E., \& Foley, J. M. (1980). Contrast masking in human vision. Journal of the Optical Society of America, 70, 1458-1471.

Legge, G. E., Kersten, D., \& Burgess, A. E. (1987). Contrast discrimination in noise. Journal of the Optical Society of America A, $\mathbf{4}$, 391-404.

LegGe, G. E., \& Viemeister, N. F. (1988). Sensory analysis in vision and audition: A commentary on Sensory analysis by Donald Laming. Behavioral \& Brain Sciences, 11, 301-302.

Leshowitz, B., Taub, H. B., \& RaAB, D. H. (1968). Visual detection of signals in the presence of continuous and pulsed backgrounds. Perception \& Psychophysics, 4, 207-213.

Levi, D. M., Jiang, B.-C., \& Klein, S. A. (1990). Spatial interval discrimination with blurred lines: Black and white are separate but not equal at multiple spatial scales. Vision Research, 30, 1735-1750.

Miller, G. A. (1947). Sensitivity to changes in the intensity of white noise and its relation to masking and loudness. Journal of the Acoustical Society of America, 19, 609-619.

Morgan, M., Chubb, C., \& Solomon, J. A. (2008). A “dipper” function for texture discrimination based on orientation variance. Journal of Vision, 8(11, Art. 9), 1-8

NACHMias, J. (1993). Masked detection of gratings: The standard model revisited. Vision Research, 33, 1359-1365.

NaChMias, J., \& SAnSbURY, R. V. (1974). Grating contrast: Discrimination may be better than detection. Vision Research, 14, 1039-1042.

Pelli, D. G. (1985). Uncertainty explains many aspects of visual contrast detection and discrimination. Journal of the Optical Society of America A, 2, 1508-1532.

Pfafflin, S. M., \& Mathews, M. V. (1962). Energy-detection model for monaural auditory detection. Journal of the Acoustical Society of America, 34, 1842-1853.

RaAB, D. H., Osman, E., \& Rich, E. (1963a). Effects of waveform correlation and signal duration on detection of noise bursts in continuous noise. Journal of the Acoustical Society of America, 35, 1942-1946.

RAAB, D. H., Osman, E., \& Rich, E. (1963b). Intensity discrimination, the "pedestal" effect, and "negative masking" with white-noise stimuli [Letter to the editor]. Journal of the Acoustical Society of America, 35, 1053.

Solomon, J. A. (2007a). Contrast discrimination: Second responses reveal the relationship between the mean and variance of visual signals. Vision Research, 47, 3247-3258.

Solomon, J. A. (2007b). Intrinsic uncertainty explains second responses. Spatial Vision, 20, 45-60.

Solomon, J. A., Felisberti, F. M., \& Morgan, M. J. (2004). Crowding and the tilt illusion: Toward a unified account. Journal of Vision, $4,500-508$

Stromeyer, C. F., III, \& KLein, S. (1974). Spatial frequency channels in human vision as asymmetric (edge) mechanisms. Vision Research, 14, 1409-1420.

Swets, J. A., Tanner, W. P., JR., \& Birdsall, T. G. (1961). Decision processes in perception. Psychological Review, 68, 301-340.

TANNER, W. P., JR. (1961). Physiological implications of psychophysical data. Annals of the New York Academy of Sciences, 89, 752-765.

Watson, C. S., Franks, J. R., \& Hood, D. C. (1972). Detection of tones in the absence of external masking noise: I. Effects of signal intensity and signal frequency. Journal of the Acoustical Society of America, 52, 633-643.

WatT, R. J., \& Morgan, M. J. (1983). The recognition and representation of edge blur: Evidence for spatial primitives in human vision. Vision Research, 23, 1465-1477.

\section{NOTES}

1. A referee thought it important that I highlight the fact that I was part of "Morgan et al." We did not fully appreciate the inelegance of our 
Figure 2 until after it had been submitted for publication. The first draft of this tutorial was submitted 4 months later.

2. I stands for intensity, which has a very precise physical definition. On the other hand, both the word "magnitude" and Weber's law are much more general.

3. $\mathrm{JND}_{\text {Fig. } 3}=\left(\mathrm{JND}_{\text {Fig. } 2}+\text { Pedestal }_{\text {Fig. } 2}\right)^{2}-$ Pedestal $_{\text {Fig. } 2^{2}}$.

4. Leshowitz, Taub, and Raab (1968), who replicated Cornsweet and Pinsker (1965), confused the terminology by introducing a hybrid paradigm, with pulsed signals of intensities $I$ and $I+\Delta I$ added to a "pedestal" of intensity $I_{\mathrm{p}}$

5. There is actually evidence for a tiny bit of negative masking in Figure 3 , since the JND for a $1-\mathrm{deg}^{2}$ pedestal is slightly less than those for the 0 - and $4-\mathrm{deg}^{2}$ pedestals.
6. Yet another quantity proportional to power.

7. Solomon (2007b) is a tutorial on signal detection theory. It includes mathematical descriptions of low-threshold theory and intrinsic uncertainty.

8. A 10-dB increase in the sound pressure level of a noise is a log-unit increase in its pressure variance. In this experiment, the same $X \mathrm{~dB}$ that were added to the mask were also added to the tone, so fluctuations in mask intensity did not affect the signal-to-noise ratio.

(Manuscript received August 20, 2008;

revision accepted for publication January 13, 2009.) 